\title{
MENINGEOMA DA GOTEIRA OLFATÓRIA
}

\author{
Osvaldo Inácio de Tella Jr', Manoel Antonio de Paiva Neto², \\ Marco Antonio Herculano 3 , Atílio Faedo Neto ${ }^{4}$
}

\begin{abstract}
RESUMO - Meningeomas da goteira olfatória representam 8-18\% dos meningeomas intracranianos e geralmente são diagnosticados quando alcançam grande tamanho. Relatamos uma série de 13 casos consecutivos de meningeomas de goteira olfativa operados nos Hospitais São Paulo (UNIFESP) e Professor Edmundo Vasconcelos no período de 1995 a 2003, estudados re trospectivamente quanto ao quadro clínico, resultados cirúrgicos e complicações. Os pacientes foram submetidos a ressecção cirúrgica destes tumores pela via subfrontal, em 9 casos a ressecção foi completa incluindo dura-máter e osso infiltrado por tumor e em 4 a dura-máter foi somente coagulada. Um paciente morreu devido a infarto cerebral e três pacientes evoluíram com fístulas liquóricas. Não houve recorrência sintomática no período que variou de 11 meses a 8 anos. Com as técnicas microcirúrgicas atuais, estes tumores podem ser removidos com baixa morbidade.
\end{abstract}

PALAVRAS-CHAVE: meningeoma, goteira olfatória, cirurgia, tratamento.

\begin{abstract}
Olfactory groove meningioma
ABSTRACT - Olfactory groove meningiomas compromise approximately $8-18 \%$ of intracranial meningiomas and harbor frequently large sizes when diagnosticated. We report 13 consecutive cases operated on Hospital São Paulo and Hospital Professor Edmundo Vasconcelos between 1995 and 2003. These patients were ret rospectively studied regarding clinical presenation, surgical results and complications. The patients und e ment uni or bifrontal craniotomies. There were nine complete resections including duramater and infiltrate bone and four patients with tumor removal and duramater coagulation. One patient died on post-operative period due to brain infarction and three patients had cerebrospinal fluid fistula that resolved. There were no symptomatic recurrence on the follow-up period of 3.3 years(range $11 \mathrm{~m}$ to 8 years). With current microsurgical techniques, these tumors can be resected safely with low morbidity.
\end{abstract}

KEY WORDS: meningioma, olfactory groove, surgery, treatment.

Meningeomas localizados na linha média da fossa craniana anterior podem ter origem de vários sítios (placa cribiforme, plano esfenoidal, tubérculo da sela e diafragma selar) e correspondem a $12-22 \%$ dos meningeomas intracranianos ${ }^{1-3}$. São convencionalmente subdivididos em dois grupos, devido apresentar sintomatologia, dificuldade e riscos cirúrgicos difere ntes. Os de goteira olfatória, quando crescem na região da placa cribiforme e plano esfenoidal (sutura frontoesfenoidal) e supra-selare $s^{3,4}$. Algumas vezes, devido ao grande tamanho que atingem quando diagnosticados, o local de inserção dural é difícil de ser diferenciado $^{3-5}$.

O desenvolvimento das técnicas de imagem, que pe rmitiram um diagnóstico mais precoce do tumor, e o aprimoramento das técnicas cirúrgicas e anesté- sicas ocorridos nas últimas décadas, levaram a queda significativa na morbidade e mortalidade associadas ao tratamento destes tumores ${ }^{6-8}$.

Relatamos os resultados cirúrgicos em uma série 13 tumores operados no Hospital São Paulo e Hospital P rofessor Edmundo Vasconcelos no período de 1995 a 2003.

\section{MÉTODO}

Entre 1995 e 2003, 13 pacientes foram submetidos a microcirurgia para ressecção de meningeoma de goteira olfatória no Serviço de Neurociru rgia da UNIFESP e do Hospital Professor Edmundo Vasconcelos. Foram excluídos os casos de recorrência e pacientes portadores de neurofibromatose. Os dados foram obtidos através da análise retrospectiva dos prontuários e dos exames radiológicos dos pacientes. Todos os pacientes foram submetidos a TC e/ou

1P rofessor Adjunto Livre Docente da Disciplina de Neurocirurgia da Universidade Federal de São Paulo e Médico Neurociru rgião do Hospital Professor Edmundo Vasconcelos, São Paulo SP, Brasil; ${ }^{2}$ Médico Neurocirurgião do Hospital São Paulo e Médico Neurocinurgião do Hospital Professor Edmundo Vasconcelos; ${ }^{3}$ P rofessor Adjunto da Disciplina de Neurocinurgia da Faculdade de Medicina de Jundiaí (Jundiaí SP, Brasil) e Médico Neurocirurgião do Hospital Professor Edmundo Vasconcelos; ${ }^{4}$ Médico Neurocirurgião do Hospital Professor Edmundo Vasconcelos.

Recebido 18 Março 2005, recebido na forma final 25 Agosto 2005. Aceito 15 Outubro 2005.

Dr. Osvaldo Inácio de Tella Jr - Rua São Paulo Antigo 145 / Bloco F / Apto 11 - 05864-010 São Paulo SP - Brasil. E-mail: detella@globo.com 
Tabela. Idade, sexo, sintomas clínicos, tamanho do tumor, invasão tumoral, tipo de ressecção, complicações e evolução dos 13 casos estudados.

\begin{tabular}{|c|c|c|c|c|c|c|c|}
\hline Caso & Idade/Sexo & Sintomas & $\begin{array}{l}\text { Tamanho } \\
(\mathrm{cm})\end{array}$ & $\begin{array}{l}\text { Invasão } \\
\text { da base }\end{array}$ & Ressecção & Complicação & Evolução \\
\hline 1 & $50 / \mathrm{M}$ & Déficit visual bilateral, anosmia & $5 \times 4 \times 3$ & Não & Simpson I & Não & Inalterado \\
\hline 2 & $23 / F$ & Diminuição visual a direita & $5 \times 4 \times 4$ & Hiperostose & Simpson I & $\begin{array}{l}\text { Fístula, } \\
\text { meningite }\end{array}$ & Melhora visual \\
\hline 3 & $66 / F$ & Alteração do comportamento & $6 \times 5 \times 4$ & Não & Simpson II & Não & Melhora \\
\hline 4 & $25 / F$ & Cefaléia & $5 \times 4 \times 3$ & Hiperostose & Simpson I & $\begin{array}{l}\text { Fístula, } \\
\text { meningite }\end{array}$ & \\
\hline 5 & $46 / F$ & Cefaléia, amaurose OE & $5 \times 5 \times 3$ & Não & Simpson I & Não & Melhora \\
\hline 6 & $60 / F$ & Alteração do comportamento & $5 \times 3 \times 4$ & Não & Simpson I & Não & Inalterado \\
\hline 7 & $51 / \mathrm{M}$ & Déficit visual bilateral & $5,3 \times 4 \times 5$ & Não & Simpson I & Não & Inalterado \\
\hline 8 & $60 / F$ & Alteração do comportamento & $5,2 \times 4 \times 4$ & Hiperostose & Simpson I & Não & Óbito \\
\hline 9 & $62 / F$ & Amaurose bilateral & $4,1 \times 3 \times 3,2$ & Não & Simpson I & Não & Inalterado \\
\hline 10 & $46 / F$ & Cefaléia, déficit visual bilateral & $5 \times 4,5 \times 4,2$ & Não & Simpson II & Não & Melhora visual \\
\hline 11 & $42 / \mathrm{M}$ & Anosmia, cefaléia & $4 \times 3,4 \times 3$ & Hiperostose & Simpson I & Fístula & Meningite \\
\hline 12 & $70 / \mathrm{M}$ & $\begin{array}{l}\text { Crise convulvisa, } \\
\text { déficit visual bilateral }\end{array}$ & $2,5 \times 3 \times 3$ & Hiperostose & Simpson II & Não & Inalterado \\
\hline 13 & $65 / F$ & Cefaléia & $4,5 \times 4 \times 3,5$ & Não & Simpson II & Não & Melhora \\
\hline
\end{tabular}

RM do encéfalo em que foram avaliadas alterações ósseas da base do crânio (presença de hiperostose), tamanho tumoral segundo os maiores diâmetros nos três eixos e invasão de seios paranasais.

Todos os pacientes foram operados por via subfrontal uni ou bilateral, sendo o grau de ressecção classificado de a cordo com escala proposta por Simpson ${ }^{9}$ e nos casos de remoção completa o assoalho frontal foi reconstrúdo utilizando pericrânio ou fascia lata. O seguimento pós-operatório variou de 10 meses a oito anos.

A Tabela mostra o resumo clínico e evolução dos casos.

\section{RESULTADOS}

Houve predomínio de pacientes do sexo feminino em relação ao masculino (9:4); a idade variou de 23 a 70 anos (média 51 anos). Os sintomaspredominantes foram: cefaléia em cinco casos, perda visual bilateral em quatro casos e unilateral em dois casos; alteração do comportamento em três casos; anosmia dois casos e crise convulsiva em um caso.

De acordo com os exames de imagem a maioria dos tumores apresentavam tamanho grande sendo o maior diâmetro superior a $4 \mathrm{~cm}$ em 12 (92\%) (Fig 1), Alterações da base do crânio foram achadas em cinco pacientes (Fig 2).

O grau de ressecção tumoral foi avaliado utilizando-se a descrição cirúrgica e exames de imagem pós-

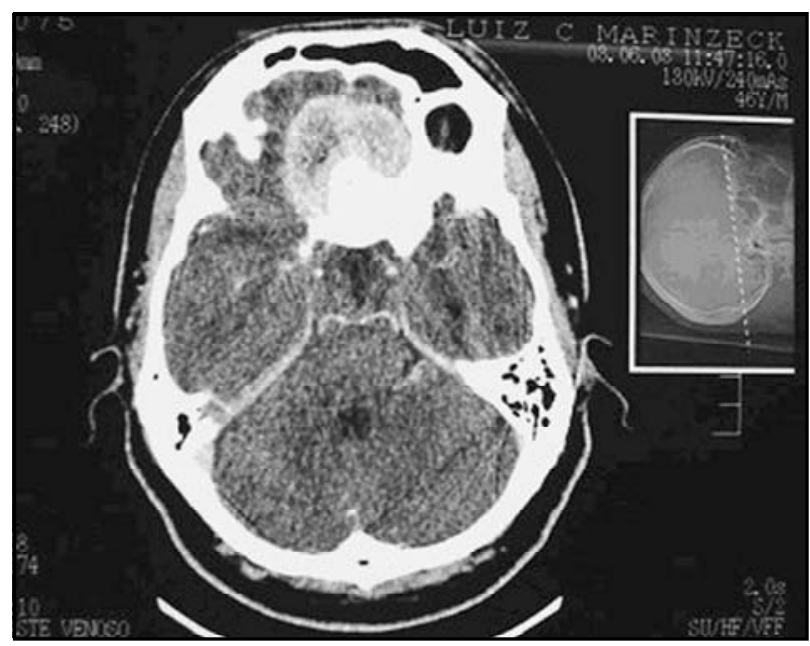

Fig 1. Tomografia axial computadorizada visibilizando hiperos tose na base do frontal.

operatória sendo classificados utilizando-se a escala de Simpson em: grau I - nove casos, grau II - quatro casos. Todos os tumores foram classificados como grau histopatológico l, utilizando-se os critérios da OMS ${ }^{10}$.

Melhora visual foi observada em dois entre seis pacientes com déficit prévio e melhora das funções cognitivas em dois casos; todos os pacientes que apresentavam cefaléia tiveram seus sintomas resolvidos. 


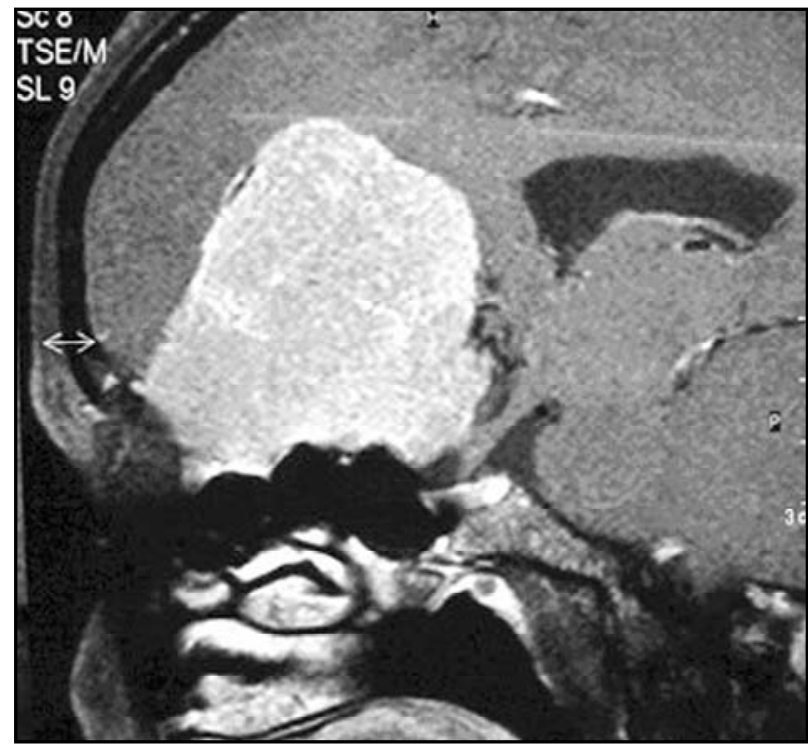

Fig 2. Ressonância magnética em corte sagital mostrando meningeoma com 4,5 cm no seu maior diâmetro.

Um paciente faleceu, no pós-operatório, devido a infarto em região das duas artérias cerebrais anteri ores, três pacientes evoluíram com fístula liquórica e meningite sendo todos tratados com derivação lombar externa e antibiótico, com resolução da fístula.

\section{DISCUSSÃO}

Meningeomas da goteira olfativa correspondem a $8-18 \%$ dos meningeomas relatados em séries cirúrgicas $5,7,11,12$. Crescem na linha média da fossa anterior deslocando os tratos olfativos lateralmente e quiasma óptico posteriormente. São nutridos primordialmente por ramos anteriores e posteriores da artéria oftálmica. O seguimento $A 2$ da artéria cerebral anterior é tipicamente deslocado posteriormente e as artérias fronto-orbitárias e fronto-polares geralmente encontram-se lateralizadas em relação ao tumor, podendo estar envolvidas no suprimento sanguíneo pial destes tumores principalmente quando atingem grande tamanho $2,3,5,7,13$.

Os meningeomas da goteira olfatória podem causar alterações hiperostóticas variáveis no plano etmoidal e esfenoidal. A presença de hiperostose na base do frontal foi relatada em 38 a $77 \%$ das séries pesquisadas na literatura ${ }^{4,7,14}$. Erosão extensa com crescimento para baixo é muito menos comum e geralmente não é uma invasão e sim destruição da base do crânio na sua região mais frágil, a lamina cribosa do etmóide; o que pode permitir a proliferação na cavidade nasal e seio maxilar e órbita ${ }^{14}$. Alguns autores relataram expansão para os seios subfrontais em até $46 \%$ dos seus casos, isto parece ser muito mais freqüente que o descrito por outros autore $s^{5,15}$. Maiuri et al. relatam um único caso em $\mathbf{4 2}$ observados no seu serv iço $^{16}$. Pieper et al. demonstraram que a hiperostose em meningeomas se deve a invasão direta do osso pelo tumor ${ }^{17}$. Em nossa casuística, cinco tumores apresentavam hiperostose basal frontal e não houve caso de invasão tumoral para os seios paranasais.

Sinais de compressão do lobo frontal, como alteração de comportamento, apatia, alteração visual, cefaléia e anosmia são os principais sintomas achados. Devido ao crescimento lento e pobreza de sintomas apresentados, a maioria destes tumores somente é diagnosticada quando atingem grandes volumes. Turazzi et al. e Hentshel tiveram mais de $50 \%$ de casos de tumores maiores que $6 \mathrm{~cm}^{5,18}$. Dolenc, em uma série de 157 tumores, relatou que 82 eram grandes (6 $\mathrm{cm})$ e 26 gigantes $(>10 \mathrm{~cm})^{14}$. Doze pacientes da nossa casuística tinham tumores maiores que $4 \mathrm{~cm}$ no maior diâmetro, sendo quatro maiores que $6 \mathrm{~cm}$.

No diagnóstico complementar a TC é import ante para delimitar as alterações ósseas associadas da base do crânio e a RM para delimitar as relações com os ne vos ópticos, hipotálamo e estruturas vasculares ${ }^{5,7,8}$.

Desde a primeira descrição de ressecção cirúrgica bem sucedida deste tumor em 1885 por Durante, dife rentes vias cirúrgicas têm sido utilizadas ${ }^{18}$. Cushing e Eisenhardt ${ }^{19}$ descreveram a craniotomia frontal unilateral com ressecção parcial do lobo frontal anterior, o que também foi recomendado posteriormente por Olive cronae Symon ${ }^{20,21}$. Turazzi et al. descrevaram a via bifrontal com amputação bilateral da ponta dos frontais ${ }^{18}$. Estes autores prese navam o seio frontal para evitar fístulas e infecção. Tonnis foi o primeiro a descrever a via frontobasal mediana com ligadura do seio sagital superior ${ }^{22}$. Seeger utilizou a subfrontal unilateral preservando o seio sagital superior ${ }^{23}$. Atualmente a craniotomia frontobasal bilateral e suas variações são as principais vias de abordagem cirúrg ica. Hassler e Zentner ${ }^{24}$, foram os primeiros a relatar a via pterional para tratamento destes tumores, posteriormente utilizada por outros autores ${ }^{18,25}$, descrevendo como vantagens desta a não elevação do lobo f rontal, evitar sacrifício das veias de drenagem frontais e visibilização mais precoce dos nervos ópticos e a rtérias do polígono de Willis. Segundo outros autores, a principal desvantagem desta via de abordagem é o manuseio da hiperostose da base do frontal e reconstrução do assoalho frontal ${ }^{5,7}$. Todos os tumores na nossa casuística foram abordados por via subfrontal uni ou bilateral, esta última sendo utilizada nos primeiros casos e nos casos de tumores de maior volume. 
Segundo Rubin, as principais causas de ressecção incompleta são o tamanho do tumor, vascularização, consistência firme, e inserção dural ampla com invasão de estruturas da base do crânio, órbitas e seio cavemoso ${ }^{2}$. Hentschel e DeMonte analisaram 106 casos da literatura e observaram que em $91 \%$ foi possível a ressecção completa 5 . Dolenc conseguiu ressecção completa em 151 dos 157 tumore $\mathrm{s}^{14}$. Na nossa casuística nove dos 13 casos obtiveram ressecção completa, incluindo o osso hiperostótico. Três casos com ressecção incompleta deveram-se a idade dos pacientes, em que foi realizada apenas coagulação da duramáter basal, com objetivo de diminuir chance de complicações pós-operatórias como fístula liquórica e/ou meningite, que não ocorreram em nenhum destes casos. No quarto caso a ressecção incompleta deveuse a escolha do cirurgião.

O tratamento de pacientes com déficit visual, mesmo de longa duração, ou com alterações do estado mental tendem a mostrar resultado satisfatório devido a grande chance de melhora visual, o que ocorreu em 2/6 pacientes de nossa série e todos na série de Turazzi et al. e Hentschel et al.5,18. A melhora cognitiva foi observada em 2/4 pacientes da nossa série e todos na de Turazzi e Hentschel. Dolenc observou melhora dos sintomas em 112 de seus 157 pacientes $^{14}$.

A principal causa de mortalidade cirúrgica é a lesão de artérias cerebrais, como carótida e cerebrais anteriore, como ocorrido em um paciente em nossa série; e ou lesão de pequenos vasos perfurantes que se dirigem ao hipotálamo que podem causar alterações diencefálicas no pós-operatório imediato como: diabetes insipidus, hiperte rmia, hiperglicemia. Nas grandes séries da literatura a mortalidade cirúrgica alcançou $33 \%$ na era pré-microscopia ${ }^{19,22}$, atualmente várias séries mostraram mortalidade de $0 \%$ 5,7,24. Outras complicações freqüentes são fístula liquórica e meningite, que são mais freqüentes quando procedimentos mais agressivos, com abertura do seio etmoidal, são utilizados durante cirurgia ${ }^{5,7,18,24}$. Fistula liquórica foi observada em três dos 13 casos da nossa série, sendo todos em paciente com ressecção Simpson I.

A cirurgia é o fator principal na prevenção de recorrência precoce e tardia destes tumores que pode variar de 0 a $41 \%{ }^{26,27}$, tendo como objetivo a remoção completa do tumor, ressecção do osso hiperostótico e remoção e coagulação da dura-máter da base frontal. Existe a discussão com o manejo da invasão óssea para prevenir a recorrência tumoral e crescimento nos seios paranasais. Apesar das ressecções amplas inclusive com etmoidectomia serem preconizadas por alguns autores ${ }^{5,7}$, outros se limitam a retirada óssea evitando a entrada no seio paranasal, julgando que o risco de fístula e infecção é maior que o de recidiva ${ }^{12,21,28,29}$. Estudos com seguimento maisprolongado são necessários para se obter esta resposta.

Al-Mefty et al. reiteram a necessidade de ressecção radical destes tumores, com retirada completa do osso hiperostótico e violação do seio etmoidal sempre que necessário, excetuando pacientes idosos, como melhor método para prevenção darecorrência ${ }^{30}$. Symon e Ojemman não observaram recorrência de meningeoma da goteira olfatória após acompanhamento médio de 4,4 anos e preconizam a ressecção óssea sem abertura do seio etmoidal, relatando ainda que as chances de complicação infecciosas são maiores que a de recorrência tumoral. Jaaskelainen estudando 657 pacientes com meningeomas em varias localizações verificou que a recorrência pode ser de até $19 \%$ em 20 anos, seus achados mostraram que a coagulação da base, invasão óssea e consistência mole do tumor são fatores de risco para recorrência ${ }^{31}$. Outros aspectos do tumor como lobulação, presença de edema e ausência de calcificação, podem ter influência na recidiva da lesão ${ }^{32}$. Também a presença de índices p roliferativos altos, como MIB-1, parece estar relacionada a recidiva tumoral maior ${ }^{5,33}$.

Não tivemos recorrência sintomática na nossa série apesar do curto seguimento, média 3,5 anos, variação de 11 meses até oito anos.

Em conclusão, com as técnicas microcirúrgicas atuais, os resultados das ressecções dos meningeomas da goteira olfatória tem sido bastante satisfatórios, com grande índice de exerese total e baixo nível de complicações. A necessidade de realização de cirurgias mais radicais com abertura do seio etmoidal para a p revenção de re co o rrência ainda é discutível, necessitando estudos com seguimentos maiores destes pacientes.

\section{REFERÊNCIAS}

1. Kallio M, Sankila L, Hakulien T, Jaaskilainen J. Factores affecting operative and excess long-term mortality in 935 pacients with intracranial meningeoma. Neurosurgery 1992;31:2-12.

2. Rubin G, David UB, Gornish M, Rappaport ZH. Meningeomas of the anterior cranial floor: review of 67 cases. Acta Neurochir (Wien) 1994;129:26-30.

3. Solero CL, Giombini S, Morello G. Suprasellar and olfactory meningioma. report of a series of 153 personal cases. Acta Neurochir (Wien) 1993;67:181-194.

4. DeMonte F. Surgical treatment of anterior basal meningiomas. J Neurooncol 1996;29:239-248.

5. Hentschel SJ, DeMonte F. Olfactory groove meningiomas. Neurosurg Focus 2003; 6:article 4.

6. Chen T, Thomas C, Zee CS, et al. Magnetic resonance imaging and pathological correlation of meningiomas. Neuro su rgery 1992;31:10151022. 
7. Obeid F, Al-Mefty O. Recurrence of olfactory groove meningioma. Neurosurgery 2003;53:534-543.

8. Suzuki $Y$, Sugimoto T, Shibuya M, Sugita K, Patel J. Meningiomas. Correlation between MRI characteristics and operative findings including consistency. Acta Neurochir (Wien) 1994;129:39-46.

9. Simpson $D$. The recu r rence of intracranial meningiomas after surgical treatment. J Neurol Neurosurg Psychiatry 1957;20:22-39.

10. Kleihues P, Burger PC, Scheithauer BW. The new WHO classification of brain tumours. Brain Pathology 1993;3:255-268.

11. ElGindi S. Olfactory groove meningiomas: surgical techniques and pitfalls. Surg Neurol 2000;54:415-417.

12. Ojeman R. Olfactory groove meningiomas. In Al-Mefty O (ed). Meningiomas. New York: Raven Press 1991:383-393.

13. McDermott M, Rootriam J, Durity FA. Subperiosteal, subperiorbital dissection and division of anterior and posterior ethmoid arteries for meningiomas of the cribriform plate and planum sphenoidale: technical note. Neurosurgery 1995;36:1215-1219.

14. Dolenc VV. Skull and skull base tumors: overview. In Winn R, Youmans (eds). Neurological surgery, Fifth edition. Philadelphia: Saunders Elsevier, 2004:1261-1281.

15. Derome PJ, Guiot G. Bone problems in meningiomas invading the base of skull. Clin Neurosurg 1978;25:431-441.

16. Maiuri F, Salzano FA, Motta S, Colella G, Sardo L. Olfactory groove meningioma with paranasal sinus e nasal cavity extension: removal by combined subfrontal and nasal approach. J Craniomaxillofac Surg 1998;26:314-317.

17. Pieper DR, Al-Mefty O, Honoda Y, Bruckner D. Hyperostosis associated changes or tumor invasion. Neurosurgery 1999;44:742-747.

18. Turazzi S, Cristofori L, Gambini R, Bricolo A. The pterional approach for microsu rgical removal of olfactory groove meningioma. Neurosurgery 1999;45:821-826.

19. Cushing H, Eisenhardt L. The olfactory meningiomas with primary anosmia. In Cushimg H, Eisenhardt L (eds). Meningiomas. Springfield: Charles \& Thomas 1938:250-282.
20. Olivercrona AH, Urban H. Uber Meningiome de Siebbierplatte. Bruns Beitr Klin Chir 1935;161:224-253.

21. Symon L. Olfactory groove and suprasellar meningiomas. In Krayenbuhl $\mathrm{H}$ (ed). Advances and technical standarts in neurosurgery. New York: Spriger-Verlag 1977:4:67-91.

22. Tonnis W. Zur Operation de Meningiome de Siebeinplatte. Zentralbl Neurochir 1938;3:1-6.

23. Seeger W. Microsurgery of the cranial base. New York: Springer Verlag 1983.

24. Hassler W, Zentner J. Pterional approach for surgical treatment of olfactory groove meningiomas. Neurosurgery 1989;25:942-947.

25. Schaller $\mathrm{C}$, Vert $\mathrm{R}$, Hassler $\mathrm{H}$. Microsurgical removal of olfactory groove meningioma via the pterional approach. Skull Base Surg 1994;4:189-192.

26. MirimanoffRO, Dosoretz DE, Lingood RM. Meningioma: analysis of recurrence and prog ression following neuro su rgical resections. J Neurosurg 1985;62:18-24.

27. Tsikoudas A, Hirsch MDP. Olfactory groove meningiomas. Clin Otolaryngol 1999;24:507-509.

28. Mathiesen T, Lindquist C, Kildstron L, Karlson B. Recurrence of cranial meningiomas. Neurosurgery 1996;39:2-9.

29. Black P. Ag ressive surgery and focal radiotherapy in the management of meningiomas of the skull base: preservation of function with maintenance of local control. Acta Neurochir (Wien) 2001;143:555-562.

30. Al-Mefty O Holoib A, Rifai A, Fox JL. Microsurgical removal of suprasellar meningiomas. Neurosurgery 1985;16:364-372.

31. Jaaskelainen J. Seemingly complete removal of histologically benign intracranial meningioma: late recur rence rate and factors predicting recurrence in 657 pacients. A multivariate analysis. Surg Neurol 1986; 26:461-464.

32. Nakasu S, Nakasu Y, Nakayama N, Mtsuda M, Hand J. Preoperative identification of meningiomas that are highly likely to recurr. J Neurosurg 1999;90:455-462.

33. Ho DM, Hsu CY, Ting LT. Histopathology and MIB-1 labeling index predict the recurrence of meningioma: a proposal of diagnostic criteria for patients with atypical meningioma. Cancer 2002;94:1538-1547. 